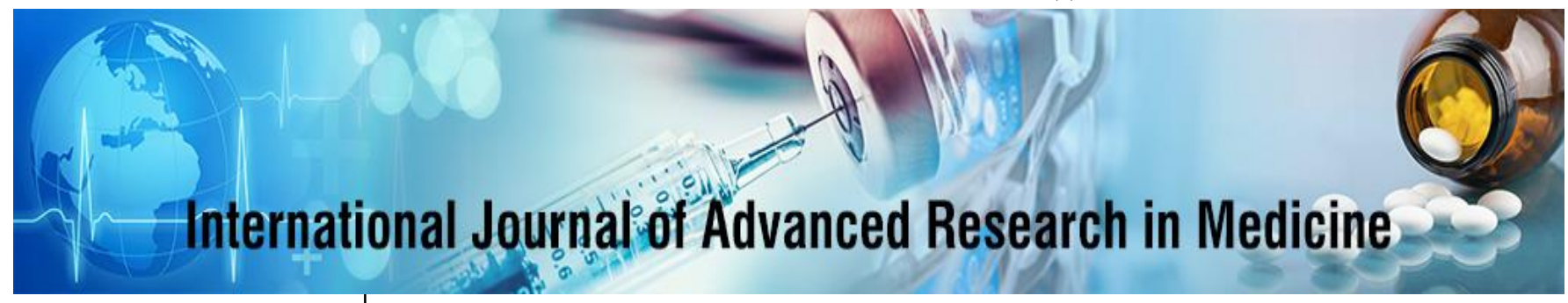

E-ISSN: 2706-9575

P-ISSN: 2706-9567

IJARM 2021; 3(2): 98-103

Received: 03-05-2021

Accepted: 07-06-2021

Sunil Kumar Sharma

Authentic Ent Head Neck

Centre, The Gujarat Research

\& Medical Institute, Rajasthan

Hospital, KD. Hospital, and

SBM Hospital. Ahmedabad,

Gujarat, India
Corresponding Author: Sunil Kumar Sharma Authentic Ent Head Neck Centre, The Gujarat Research \& Medical Institute, Rajasthan Hospital, KD. Hospital, and SBM Hospital. Ahmedabad, Gujarat, India

\section{Case series of rhinocerebral mucormycosis second wave covid 2021: Mortality or survival real factors}

\author{
Sunil Kumar Sharma
}

DOI: https://doi.org/10.22271/27069567.2021.v3.i2b.224

\begin{abstract}
Background: Rhinocerebral mucormycosis is a complex and fatal infection typically affecting diabetic and/ or immunocompromised patients, but recently seen in large numbers during the second wave of corona infection. This dreadly infection was found typically in patients who were administered steroids and higher antibiotics. Usually, these kinds of manifestations are noticed due to inhalation of fungal spores. Fungi constitute a kingdom of ubiquitous eukaryotic organisms that actively replicate during decomposition and recycling of matter in the natural environment. About 50000 fungal species, of which only 400 are actually pathogenic for humans. Approximately a dozen of which are responsible for $90 \%$ of frequently encountered fungal infections.

Material and methods: Survival rate of patients is moderately high 95\% and Mortality rate is only 5\% in our series due to early diagnosis and treatment with aggressive sugar control, repeated debridement antifungal therapy and regular follow up with MRI PNS Contrast.

Results: In this case series, 156 cases with rhinocerebral mucormycosis were presented. The etiologic agents and other survival and Mortality factors of mucormycosis in 156 Patients were isolated and identified by sequence analysis and data were registered and presented.

Conclusion: In patients with mucormycosis, early detection, surgical excision and appropriate debridement, suitable antifungal therapy, MRI Contrast and control of risk factors like, Covid patient on steroids and higher antibiotics, diabetes mellitus are the main parameters of successful management of this lethal infection.
\end{abstract}

Keywords: rhinocerebral mucormycosis, diabetes mellitus, antifungal therapy. MRI contrast

\section{Introduction}

In Covid Era After April 4, 2021 surge in high number of cases of corona, after 15 days of covid hike there are high number of Mucormycosis cases emerges in India in huge numbers. mainly high dose of steroids, diabetes, higher antibiotics, iron and Zinc supplement and virus itself leading to destruction of CD8 cytotoxic cells.

Rhinocerebral mucormycosis is an acute and often lethal opportunistic fungal infection typically affecting diabetic ( $85 \%$ of the cases) or immunocompromised patients

caused by fungi of the class zygomycetes ${ }^{[1]}$. Without early treatment, rapid progression of disease with 50 to $80 \%$ mortality rates from intraorbital and intracranial complications have been reported ${ }^{[2]}$. Our case series have described the mortality of approximately $5 \%$ in rhinocerebral mucormycosis. The infection has high incidence in diabetic patients due to the greater availability of glucose to the pathogen, lower response of

T-cells, reduced serum inhibitory activity against the Rhizopus in lower $\mathrm{pH}$, and increased expression of some host receptors that mediate the invasion of human epithelial cells through microorganism ${ }^{[3]}$. The aim of this case series was to present patients with rhinocerebral mucormycosis who were successfully treated due to timely diagnosis of infection, and identification of etiologic agent by $\mathrm{KOH} / \mathrm{GMS}$ fungal Preparation, fungal culture and histopathology examination of nasal tissues ${ }^{[4]}$.

\section{Pathogenesis}

The infection usually presents as post covid history mainly after 15 days of covid diagnosis with higher steroids, DM with ketoacidosis. Acute sinusitis with fever, nasal congestion, discharge, and headache sinus pain and facial numbness ${ }^{[2]}$. All of the sinuses become involved and spread to the contiguous structures, such as the palate orbit and brain, usually progress rapidly. 
The hallmark of spread beyond the sinuses are tissue necrosis of the palate, resulting in palatal eschars, destruction of the turbinates, perinasal swelling, erythema, cyanosis of the facial skin and overlying tissues. Signs of orbital involvement include

periorbital edema, proptosis and blindness [1]. Facial numbness is frequent and results from infarction of sensory branches of the fifth cranial nerve ${ }^{[2]}$.

Triad of 1-Covid patient on steroids, 2-diabetes with headache / facial pain with 3-abnormal MRI Mostly Mucormycosis until Prove otherwise.

The common underlying diseases

- Post covid after steroids

- Higher steroids

- Iron and Zinc supplements

- Use of higher steroids

- DM with ketoacidosis,

- Patients On Steroids

- Malignancies

- Solid organ transplant

- iron overload

- AIDS

- Treatment with deferoxamine

- Drug abuse

- Trauma/Burns

- Malnutrition

Rhizopus organism has an enzyme, ketone reductase that allows them to thrive in high glucose, acidic conditions. Necrosis and infarction of infected tissues is a hallmark of mucormycosis. Mucorales hyphae are broad 2 to 5 microns, irregularly branched and without septation ${ }^{[6]}$.

\section{Treatment protocol}

our protocol to treat mucormycosis management include

1. Control of underlying pathology like sugar control, diabetic care and addressing other Haematological factors.

2. Better rehydration, Infused normal saline $60 \mathrm{ml}$ per hour to prevent renal shutdown due to amphotericin.

3. Assessment of the fungal extension and staging mainly by Mri Contrast, Nasal/Sinus Endoscopy and planning of debridement and surgery.

4. Antifungal Therapy In current practice, amphotericin is the sole antifungal agent licensed by the US Food and Drug Administration. Antifungal treatment options consist of lipid formulations of amphotericin B, amphotericin B deoxycholate, or posaconazole ${ }^{[7]}$. Firstline treatment is with an amphotericin derivative, preferably with Liposomal Amphotericin B. Liposomal Amphotericin B has proven efficacy in the treatment of mucormycosis. At the present time, the liposomal formulation is the drug of choice based on efficacy and safety data. Lipid preparations

of amphotericin B are used at $5 \mathrm{mg} / \mathrm{kg} / \mathrm{d}$. Some have used doses of up to $7.5-10 \mathrm{mg} / \mathrm{kg} / \mathrm{d}$ to treat mucormycosis, especially CNS disease; however, the benefit of higher doses is unknown and these doses have a higher risk for nephrotoxicity ${ }^{[8]}$. Amphotericin B deoxycholate can also be used for the treatment of Mucormycosis, especially in settings of cost restraints. The typical dose is $1-1.5 \mathrm{mg} / \mathrm{kg} / \mathrm{d}$. The total dose given over the course of therapy is usually

2.5-3 g. High doses of this drug are required, and nephrotoxicity may result. This is of particular concern since many patients who develop mucormycosis have pre existing renal disease. Monitor the renal function of patients taking amphotericin B doubling of serum creatinine over the baseline levels is an indication for changing to liposomal amphotericin B In addition, careful monitoring and repletion of serum electrolytes (e.g phosphorus, potassium and magnesium) should be performed when administering any formulation of amphotericin B. Posaconazole

Posaconazole, a triazole, is currently considered a second-line drug for treatment of mucormycosis and the typical dose is 5-6 ml qid in oral syrup and oral tablets [100 mg 3-0- 3 on day one and 0-3-0 second day onwards ]as available in India (total of 800 $\mathrm{mg} / \mathrm{d}$ ).Administration with a high-fat meal/food and acidic beverages enhances absorption of the drug Patients on posaconazole should avoid antacids, especially proton pump inhibitors. In Addition, therapeutic drug monitoring of posaconazole levels should be considered in patients at high risk.

5. Aggressive surgical debridement of necrotic tissue in combination with medical therapy is mandatory for patient survival. In rhinocerebral disease, surgical care includes sinus, nasal surgery and may require excision of the orbital contents and involved brain. Repeated surgery may be required.

\section{Materials and Methods}

We are presenting details of our patients which were treated in various hospitals of Ahmedabad, authentic ENT Centre, The Gujarat Research \& Medical Institute, K.D. Hospital, Sbm Hospital, during the Period of April 2021to JUNE 2021 (3 Months Period during Covid Surge).

We included several factors and recorded all details of our patients.

Total Patients: 156 All Patients Are Post Covid Mucormycosis

Male: Female $=86: 70$

Age Groups $>50$ YRS $=110<50$ YRS=46

A: Immunocompromised: 154

Mainly Dibetic 154

B: immunocompetent Non Diabetes 2

Total survival $=149$ total death $=7$

\begin{tabular}{|c|c|}
\hline Intervention within 72 hours & Survival $>99 \%$ \\
\hline Intervention after 72 hours & Mortality $>20 \%$ \\
\hline
\end{tabular}

So factors affecting the survival are

1. timing of presentation $<72$ hours

2. timing of intervention within 6hours of admission

3. severity of diabetic ketoacidosis

4. status of immunity immunocompetent

5. Debridement extension

6. Extension of fungal involvement

7. CNS involvement

8. Age of presentation younger recover early

9. Antifungal therapy and monitoring

We found that patient who diagnosed and treated early mortality was very less even in severe cases. Definitive diagnosis should be made by clinical manifestation of the 
disease, histopathological examination of infected tissues, culture (culture studies are usually unsuccessful), and radiographic features. In most of cases MRI of the paranasal sinuses (PNS) was performed.

Hypointense areas with perisinus in flammation. T1 weighted central areas of hypointensity with peripheral enhancement, T2 weighted central areas of hypointensity or signal void with peripheral enhancement ${ }^{[2]}$. There was evidence of air fluid level in sinuses compatible with acute sinusitis. There is evidence of enhanceable soft tissues density in maxillary sinus, ethmoidal air cell with bone destruction and extension to and retro orbital and intracranial area. Histopathological findings and the results of culture confirmed our diagnosis. The isolate was identified as Mucor, Rhizopus, Rhizomucor, Absidia and cunninghamella.

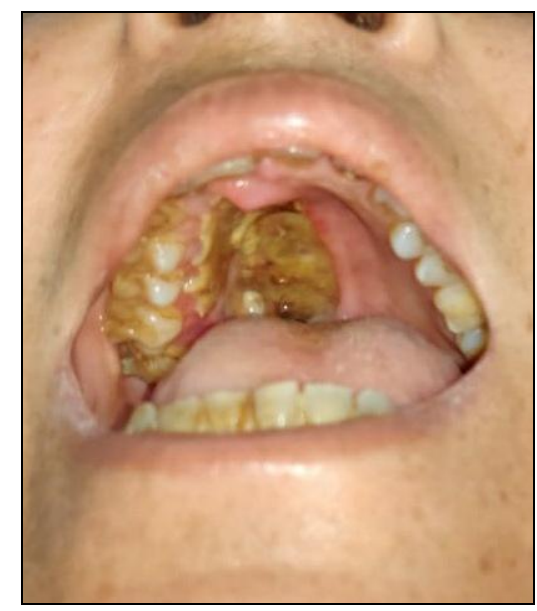

Fig 1: typical palatal eschar

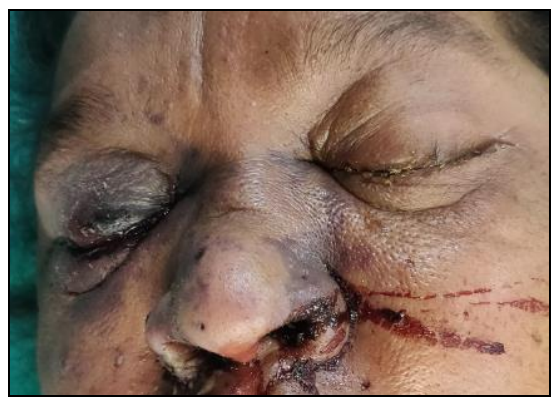

Fig 2: extensive disease

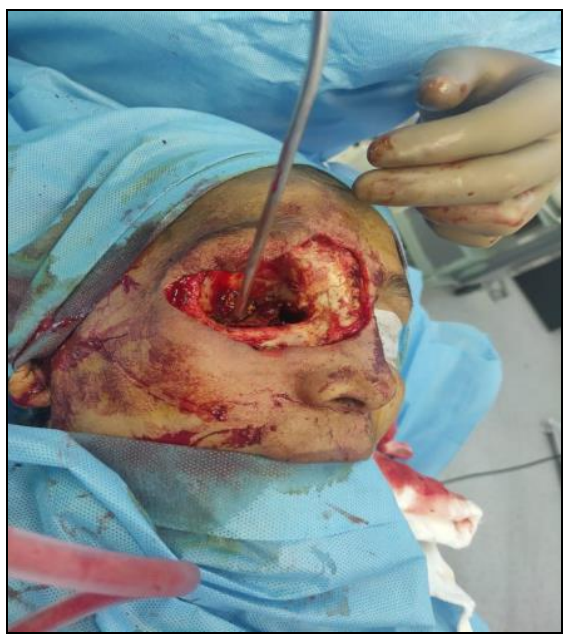

Fig 3: Extensive tissue debridement with orbital Exenteration

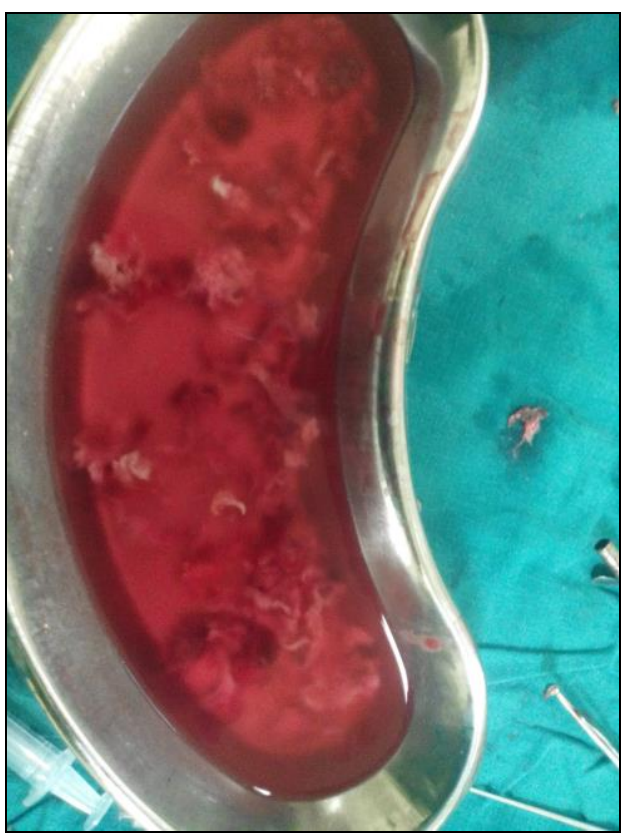

Fig 4: Extensive fungal gross tissue

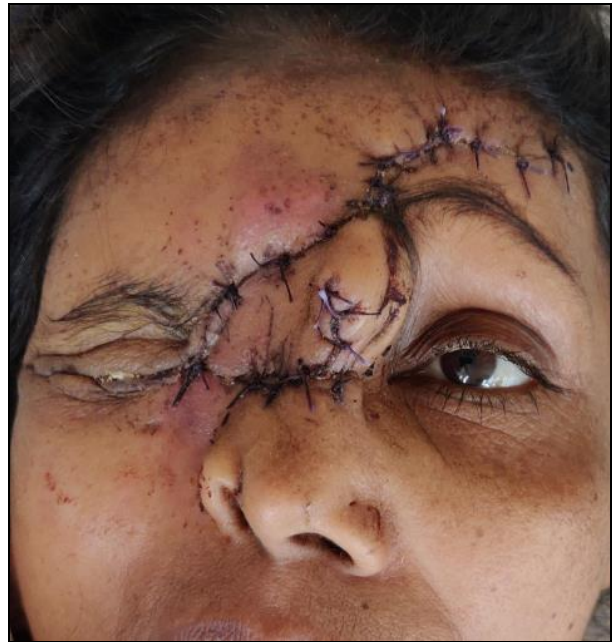

Fig 5: Post Op Reconstruction of defect with local forehead flap

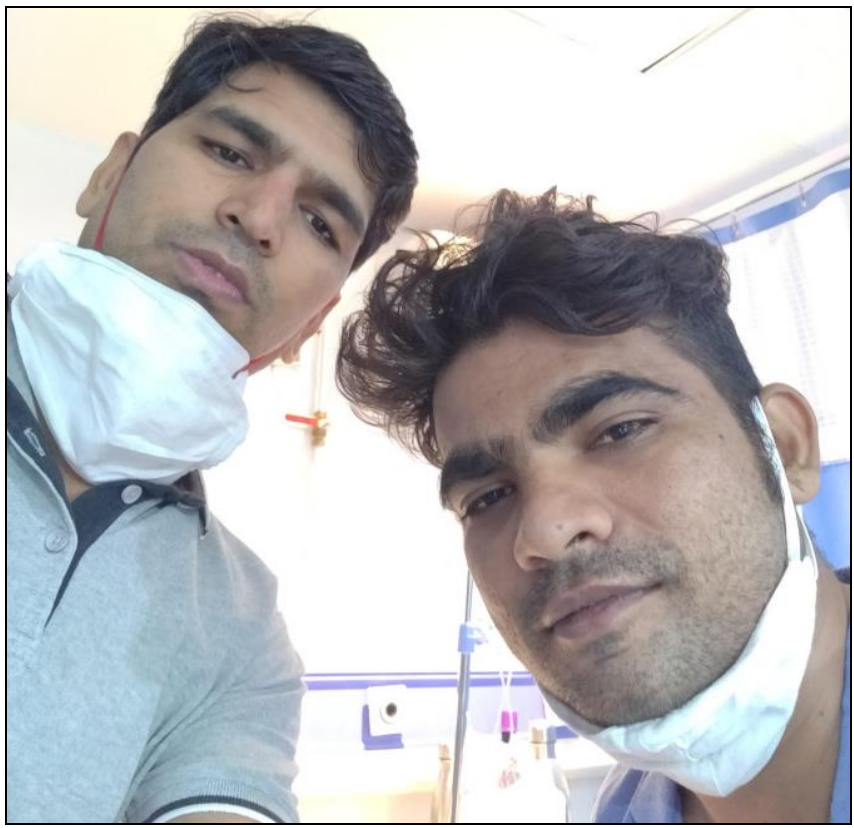

Fig 6: post op Patient sitting 


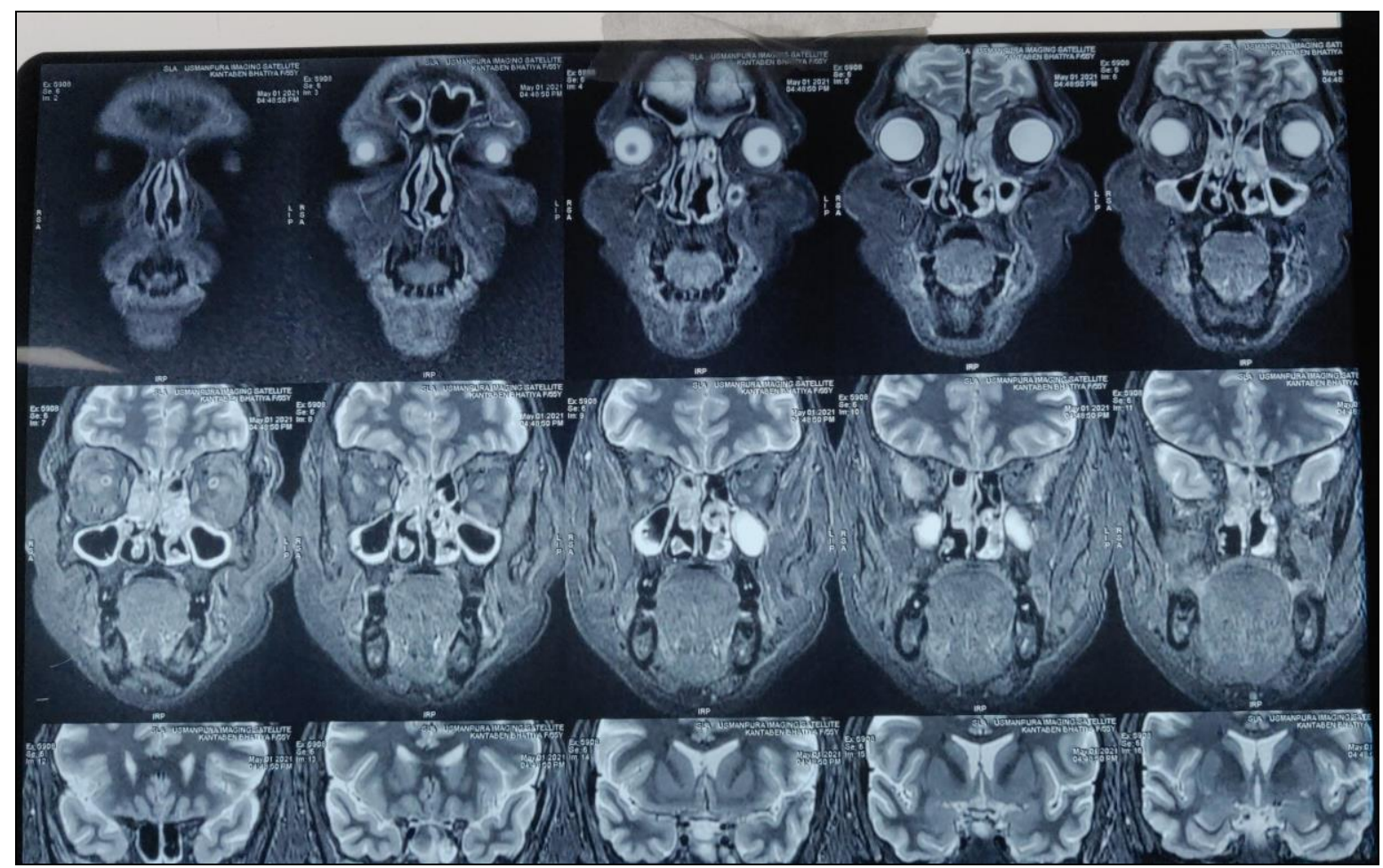

Fig 7: Extensive fungal sinusitis

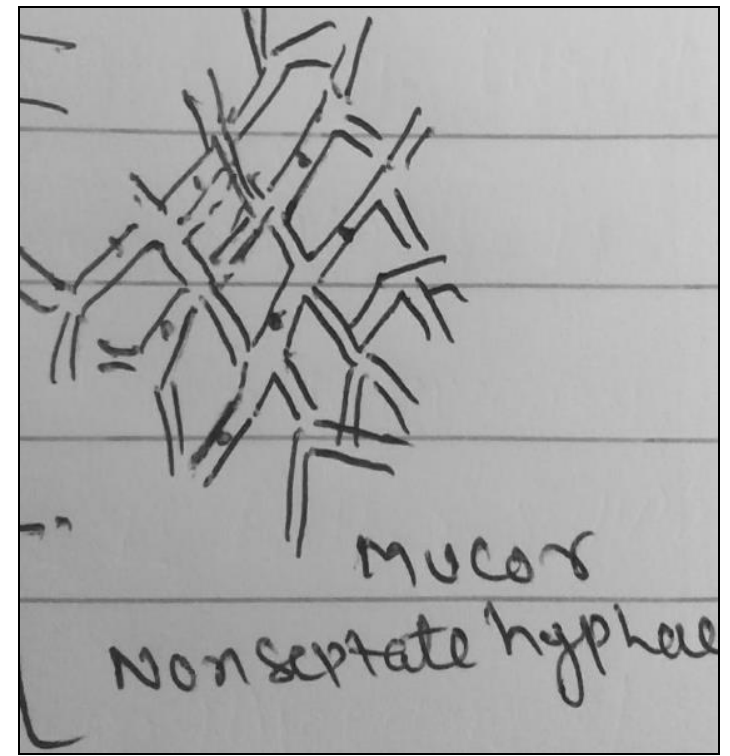

Fig 8: Mucor typical hyphae drawing

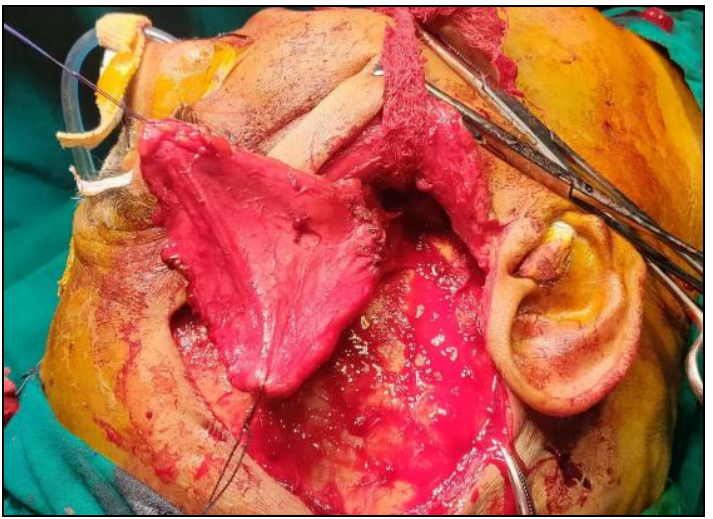

Fig 10: Palatal defect reconstruction by Temporalis muscle flap.

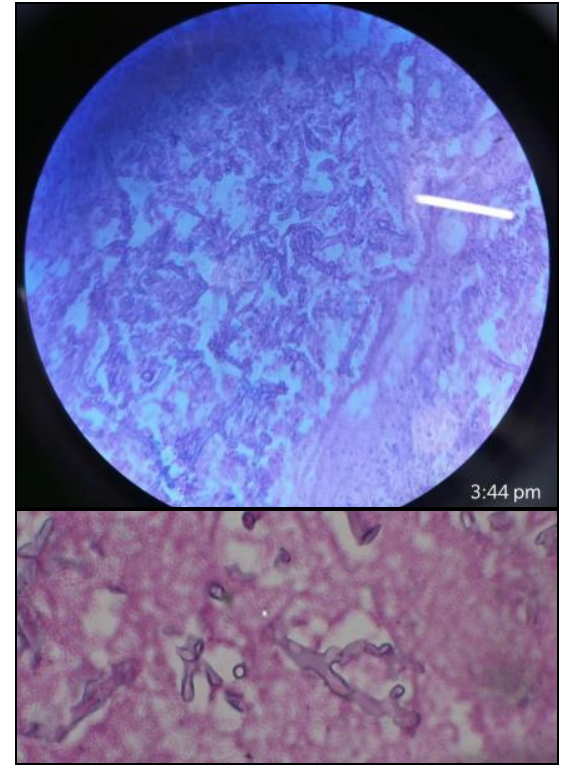

Fig 9: HPE MUCOR. (Courtesy by Dr. Parul Dargar senior pathologist)

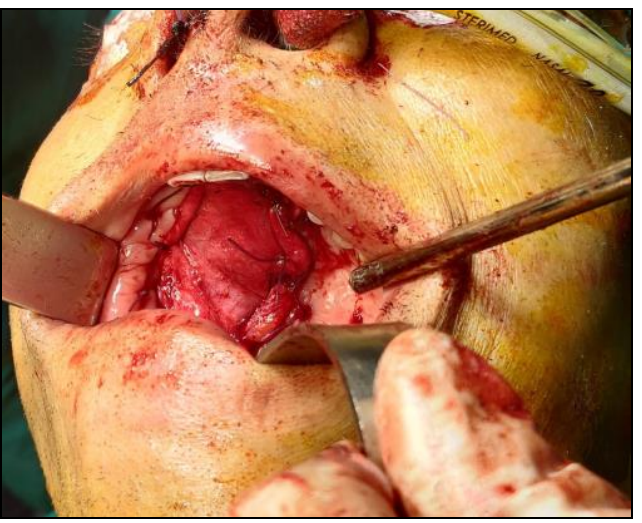




\section{Results}

Mortality rate of mucormycosis is less in our series due to early intervention, repeated debridement and aggressive antifungal therapy. In most of cases we did 3 repeated and regular debridement and aggressive diabetic control with 24 hour hydration. In most of cases we used conventional amphotericin -B for 15 to 30 days, outcome was very nice, no Patient develop Renal shutdown. We were monitoring therapy with daily serum potassium and serum creatinine. Most of the patients died due to brain infarct due to fungal vascular invasion and direct brain tissue involvement, these all Patients were delayed present to us. After 15 days liposomal/conventional amphotericin -B after that we used syrup or tablet posaconazole, no difference in outcome. In 30 patients who had Renal problems we used liposomal amphotericin -B, then posaconazole.

\begin{tabular}{|c|c|}
\hline 35 cases & Amphotericin -B for 30-40 days \\
\hline 121 cases & Amphotericin -B /liposomal for 15 days then syrup posaconazole \\
\hline
\end{tabular}

Most patients were above 50 yrs of the age and male were more affected. Most of are patients are diabetic mainly due to covid steroids use. We had 2 patients who are immunocompetent. Most patients with mucor present with high sugar levels and high total counts. We did early endoscopic debridement, partial maxillectomy, orbital decompression and orbital exenteration according to the stage of presentation. We did reconstruction after 1 month of disease control, in 3 cases we did local flap reconstruction in eye Exenteration cases and partial maxillectomy cases.

CNS involvement with frontal lobe and cavernous sinus:-7

Orbital involvement with vision loss: 10

Orbital involvement with normal vision: 2

Without any orbital involvement//pure pansinusitis: 144 Cases

We staged our cases and suggested this classification by. Dr. Sunil Kumar Sharma, consultant Department of otorhinolaryngology and head $\&$ neck surgery

\begin{tabular}{|c|c|c|c|}
\hline Stage & Subtype and details & Surgeries & Mortality \\
\hline 1 & $\begin{array}{c}\text { A. Unilateral sinusitis, } \\
\text { B. Bilateral sinusitis }\end{array}$ & Endoscopic debridement & $1 \%$ \\
\hline 2 & $\begin{array}{c}\text { A. Palatal involvement<2 cm } \\
\text { B. Palatal involvement>2 Cm }\end{array}$ & $\begin{array}{c}\text { A. Endoscopic debridement with palate debridement } \\
\text { B. Endoscopic debridement with Partial maxillectomy }\end{array}$ & $3 \%$ \\
\hline 3 & $\begin{array}{c}\text { A. orbital involvement with normal vision } \\
\text { B. orbital involvement with vision loss }\end{array}$ & $\begin{array}{c}\text { A. Endoscopic debridement with orbital decompression } \\
\text { B. Endoscopic debridement with orbital Exenteration }\end{array}$ & $10 \%$ \\
\hline 4 & $\begin{array}{c}\text { A. CNS involvement with consciousness } \\
\text { B. CNS involvement with loss of consciousness } \\
\text { C. facial skin involvement }\end{array}$ & $\begin{array}{c}\text { Endoscopic Resection with involvement brain tissue and } \\
\text { external skin resection }\end{array}$ & $40 \%$ \\
\hline
\end{tabular}

\section{Discussion}

Mucormycosis is a fungal infection caused by a member of the family Mucoraceae. Rhizopus, Mucor, Absidia are the most common isolated from patients with mucormycosis. Rhizopus is responsible for $60 \%$ of all cases of mucormycosis, and $90 \%$ of rhinocerebral mucormycosis ${ }^{[9]}$. The fungi are found in soil, bread, mold, rotten fruits and vegetables. The most common and fatal is rhinocerebral involvement. Though mucormycosis is ubiquitous and grows rapidly, it seldom strikes in immunologically competent patients. Therefore, if an infection with mucormycosis occurs, it usually indicates a serious underlying medical condition. Because the disease provokes diffuse tissue necrosis, the fungi can easily invade the wall of blood vessels, leading to thrombosis and tissue ischemia [10]. Therefore, it is not uncommon to find the infection spreading to the cavernous sinus or the central nervous system. The deterioration in mental status is an ominous sign, often heralding intracerebral extension of the disease process. All of these symptoms may develop over a period of several days or may occur as a fulminating process within hours. Imaging studies are important to evaluate the extent of the disease. MRI of patients with rhinocerebral mucormycosis shows opacification of the paranasal sinus, Hypointense area with perisinus inflammation. There are thickening of the sinus mucosa and bone destruction without an air-fluid level ${ }^{[11]}$. In addition, when the orbit is invaded, increased density of the orbital fat and venous engorgement may be seen. Magnetic resonance imaging (MRI) can demonstrate soft tissue lesions better, especially in diagnosis of cavernous sinus thrombosis.

In the early stages, nasal endoscopic findings may be as subtle as the presenting symptoms. Alteration in mucosal appearance such as a discoloration, granulation ulceration, black eschar, and black crusting are the most consistent physical findings ${ }^{[2]}$.

Biopsy of the affected tissue is required to confirm the infection. In the histologic section, these organisms are characterized by wide, non-septate hyphae with right-angled branching. Cultures are still the standard means of diagnosis. But even positive histologic findings, routine sinus cultures and blood cultures are rarely positive ${ }^{[12]}$. Treatment of rhinocerebral mucormycosis should consist of prompt control of hyperglycemia and ketoacidosis, aggressive surgical debridement of involved tissue, and administration of parenteral amphotericin B. The importance of surgery is pronounced when no surgical treatment or only biopsy was performed the mortality rate is very high, which seen in Patient who refused to operate.

\section{Conclusion}

Rhinocerebral mucormycosis is an acute opportunistic mycosis that predominantly occurs in the patients with diabetes. The physician may see patients with RCM in its earliest stages masquerading as other less serious diseases. Early diagnosis aggressive surgical debridement, high dose amphotericin B and good control of blood sugar are the most important factors to decrease the morbidity and 
mortality from this fungal disease. Early detection, surgical excision and appropriate debridement, suitable antifungal therapy, and control of risk factors like diabetes mellitus are the main parameters of successful management of this lethal infection among diabetic patients.

Video links:-https://youtu.be/JM3aqBdWCQI

https://youtu.be/PJrVMmlgRRo,

\section{Acknowledgments}

The authors express their appreciation to Authentic Ent Head and Neck Centre, The Gujarat research \& medical institute Rajasthan Hospital personnel, K.D. Hospital, Sbm Hospital

Conflict of interest: The authors express no conflict of interest.

\section{References}

1. Sataloff's comprehensive textbook of otolaryngology, head \& neck surgery, rhinology/allergy and immunology first edition 2016;2:451-465.

2. Scott-brown's otorhinolaryngology head \& neck surgery rhinology eight edition 2019;1:1047-1057.

3. Cummings otolaryngology head \& neck surgery, $5^{\text {th }}$ edition 2010;48(1):710-12.

4. Ricardo AR, Hector RM, Coronado $\mathrm{S}$ et al. Rhinocerebral and systemic mucormycosis clinical experience with 36 cases. J Neurol Sci 1996;143:19-30.

5. Vijayabala GS, Annigeri RG, Sudarshan R. Mucormycosis in a diabetic ketoacidosis patient. Asian Pac J Trop Biomed 2013;3:830-3.

6. Herrera DA, Dublin AB, Ormsby EL, Aminpour S, Howell LP. Imaging Findings of Rhinocerebral Mucormycosis. Skull Base 2009;19:117-25.

7. Casqueiro $\mathrm{J}$, Casqueiro $\mathrm{J}$, Alves $\mathrm{C}$. Infections in patients with diabetes mellitus: A review of pathogenesis. Indian J Endocrinol Metab 2012;16:S2736.

8. Atkins RC, Zimmet P. Diabetic kidney disease: Act now or pay later. Saudi J Kidney Dis Transpl 2010;21:217-21.

9. Peleg AY, Weerarathna T, McCarthy JS, Davis TM. Common infections in diabetes: Pathogenesis, management and relationship to glycaemic control. Diabetes Metab Res Rev 2007;23:3-13.

10. Petrikkos G, Skiada A, Lortholary O et al. Epidemiology and clinical manifestations of mucormycosis. Clin Infect Dis 2012;54:S23-34.

11. Mohammadi S, Daneshi A, Javadi M. Orbitorhinocereberal: report of 9 cases. RJMS 2002;8:397-407.

12. Tavanaee Sani A, Fata A, Arian M. Presenting features and outcome of rhino-orbital-cerebral mucormycosis in two referral center in Mashhad. Tehran Univ Med J 2014;72:46-51. 